\title{
Slow light propagation and amplification via electromagnetically induced transparency and four-wave mixing in an optically dense atomic vapor
}

\author{
Nathaniel B. Phillips, ${ }^{1}$ Alexey V. Gorshkov, ${ }^{2}$ and Irina Novikova ${ }^{1}$ \\ ${ }^{1}$ Department of Physics, College of William and Mary, Williamsburg, Virginia 23185, USA \\ ${ }^{2}$ Department of Physics, Harvard University, Cambridge, Massachusetts 02138, USA
}

(Dated: March 1, 2022)

\begin{abstract}
We experimentally and theoretically analyze the propagation of weak signal field pulses under the conditions of electromagnetically induced transparency (EIT) in hot Rb vapor, and study the effects of resonant four-wave mixing (FWM). In particular, we demonstrate that in a double- $\Lambda$ system, formed by the strong control field with the weak resonant signal and a far-detuned Stokes field, both continuous-wave spectra and pulse propagation dynamics for the signal field depend strongly on the amplitude of the seeded Stokes field, and the effect is enhanced in optically dense atomic medium. We also show that the theory describing the coupled propagation of the signal and Stokes fields is in good agreement with the experimental observations.
\end{abstract}

PACS numbers: 42.50.Gy, 32.70.Jz, 42.50.Md

\section{INTRODUCTION}

In recent years, electromagnetically induced transparency (EIT) 1, 2 and the associated effect of ultraslow pulse propagation ("slow light") [3] have attracted considerable attention due to their many promising applications. For example, they enable coherent, reversible transfer between the quantum states of an electromagnetic field and the collective excitation of an ensemble of long-lived radiators (e.g., spins of atoms, solid-state impurities, quantum nanostructures, etc.), which are necessary for the realization of quantum memory [4, entanglement of distant matter nodes [5], single photon generation [6], and realization of deterministic two-qubit gates for photons [7, etc. At the same time, more robust control of the propagation of classical pulses using EIT has been extensively explored in various materials for applications such as optical packet switching and optical signal processing [8].

In a traditional EIT scheme, a strong classical control field is applied to one optical transition, resulting in a modification of the optical properties of a weak signal field, which couples the same excited electronic state with a second long-lived ground-state sublevel, thereby forming a $\Lambda$ system [1, as depicted in Fig. 1(a). In the limit of low optical depth, it is sufficient to take into account only the effects of this single $\Lambda$. However, many applications require operation at high optical depth [9, 10, where additional nonlinear effects may become important 11, 12, 13, 14. One such effect is resonant fourwave mixing - a nonlinear process arising from the far off-resonant interaction of the control field. Earlier studies [15, 16] found that the propagation of the signal field in this case will be strongly affected by the presence of the Stokes field.

The effect of four-wave mixing can be advantageous or detrimental, depending on the details of the application. For example, in quantum memory applications the resonant mixing reduces the fidelity by adding extra

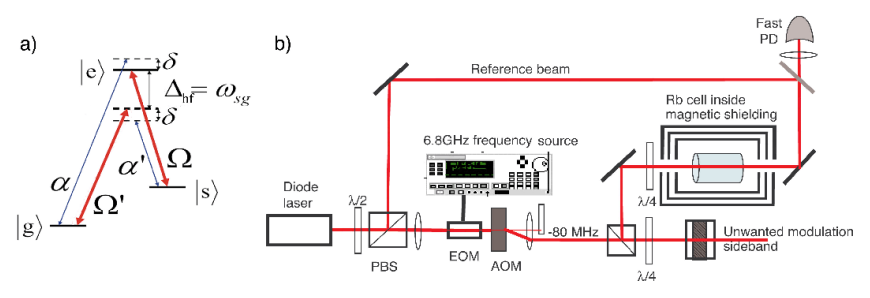

FIG. 1: (Color Online) (a) The double- $\Lambda$ system used in theoretical calculations. (b) A schematic of the experimental apparatus (see text for abbreviations).

noise into the signal field. Also, FWM may limit the storage efficiency at higher optical depth [17]. On the other hand, non-classical correlations between two signal and Stokes fields can individually carry quantum information [18 and produce entangled images. Similarly, for slow light applications, the conversion of an original pulse from the signal to Stokes channel may reduce the readout efficiency [19. However, under certain conditions, FWM may lead to gain for both the signal and Stokes fields, which could compensate for any optical losses 20 .

In this manuscript, we investigate the modification of the two-photon resonant transmission peak and the dynamics of pulse propagation in the case of a seeded Stokes field, which was produced simultaneously with the signal field by phase modulating the original monochromatic control field. Specifically, we show that the resulting signal and Stokes spectra are well-described by a double- $\Lambda$ system. Additionally, we show that this model accurately portrays the dynamics of signal and Stokes pulse propagation.

\section{EXPERIMENTAL ARRANGEMENTS}

Measurements were performed using the configuration in Fig. 1.(b). We tuned a commercial external cavity 
diode laser (ECDL) near the Rubidium $D_{1}$ transition $(\lambda=795 \mathrm{~nm})$. After using a polarizing beam splitter (PBS) to separate a fraction of the light for a reference beam, we passed the main beam through an electrooptical modulator (EOM), which modulated its phase at the frequency of the ground state hyperfine splitting of ${ }^{87} \mathrm{Rb}\left(\Delta_{\mathrm{hf}} / 2 \pi=6.835 \mathrm{GHz}\right)$. Due to the small driving amplitude, the phase modulation mostly produced two first modulation sidebands at $\pm \Delta_{\mathrm{hf}}$ of nearly equal amplitudes and opposite phases. We tuned the zeroth order (carrier frequency) field to the $5^{2} \mathrm{~S}_{1 / 2} \mathrm{~F}=2 \rightarrow$ $5^{2} \mathrm{P}_{1 / 2} \mathrm{~F}^{\prime}=2$ transition; this beam acted as the control field. The +1 modulation sideband of the amplitude functioned as the signal field, and was tuned near the $5^{2} \mathrm{~S}_{1 / 2} \mathrm{~F}=1 \rightarrow 5^{2} \mathrm{P}_{1 / 2} \mathrm{~F}=2$ transition. The -1 sideband acted as the far-detuned Stokes field. Then all optical fields passed through an acousto-optical modulator (AOM) operating at $80 \mathrm{MHz}$, which shifted the frequencies of the fields by that amount.

For spectral measurements, the control field was always on, thereby ensuring that most of the atoms were in $|g\rangle$, and we slowly swept modulation frequency of the EOM, which synchronously scanned the two-photon detuning for the Stokes and signal fields. For slow light measurements, we first applied a pulse of the control field to optically pump the atoms into $|\mathrm{g}\rangle$, and then we adjusted the modulation power of the EOM and AOM to produce desired Gaussian pulses of the signal and Stokes fields.

To carefully evaluate the effects of resonant four-wave mixing, we used a temperature tunable Fabry-Pérot etalon $(\mathrm{FSR}=20 \mathrm{GHz}$, finesse $\approx 100)$ to reduce the Stokes field amplitude by tuning the etalon transmission resonance such that most of the -1 sideband is transmitted, but all the other fields are reflected. This way, we were able to reduce the intensity of the Stokes field by a factor of 20 without noticeable losses in the intensity of both the control and signal fields.

Before entering a vapor cell, the beam was weakly focused to either $2.6 \mathrm{~mm}$ or $4 \mathrm{~mm}$ diameter, as we indicate below, and circularly polarized with a quarter-wave plate $(\lambda / 4)$. Typical peak control field and signal field powers were approximately $19 \mathrm{~mW}$ and $50 \mu \mathrm{W}$, respectively. A cylindrical Pyrex cell, of length $75 \mathrm{~mm}$ and diameter 22 $\mathrm{mm}$, contained isotopically enriched ${ }^{87} \mathrm{Rb}$ and 30 Torr $\mathrm{Ne}$ buffer gas, so that the pressure-broadened optical transition linewidth was $2 \gamma=2 \pi \times 290 \mathrm{MHz}$ 21. The cell was mounted inside tri-layer magnetic shielding, as to reduce the effects of stray magnetic fields. The temperature of the cell (and thus the concentration of $\mathrm{Rb}$ in the vapor phase) was adjusted using a bifilar resistive heater wound around the innermost magnetic shielding layer in the range between $70^{\circ} \mathrm{C}$ and $80^{\circ} \mathrm{C}$, which corresponded to the change in $\mathrm{Rb}$ densities from $5.6 \times 10^{11} \mathrm{~cm}^{-3}$ and $1.2 \times 10^{12} \mathrm{~cm}^{-3}$, and to the range of optical depths $2 d$ between 52 and 110 . Here we define the optical depth $2 d$ such that the probe intensity without EIT is attenuated by $\mathrm{e}^{-2 d}$, and our procedure for calculating the ef- fective optical depth is described in Ref. [17. We also measured [17. the typical spin wave decay time to be $1 /\left(2 \gamma_{s g}\right) \simeq 500 \mu \mathrm{s}$, most likely arising from small, uncompensated remnant magnetic fields.

After the cell, the output laser fields were recombined with the unshifted reference beam at a fast photodetector, and the beat note signals between each of the +1 and -1 modulation sidebands and the reference field was measured using a microwave spectrum analyzer. Because of the $80 \mathrm{MHz}$ frequency shift introduced by the AOM, the different beat note frequencies of each sideband with the reference field allowed for independent measurement of their amplitudes.

\section{THEORETICAL MODEL}

In a traditional three-level system, under the conditions of electromagnetically induced transparency, a strong control field (frequency $\nu_{\mathrm{C}}=\omega_{e s}$, Rabi frequency $\Omega)$ 22 works in conjunction with a weak signal field $(\nu=$ $\omega_{e g}+\delta$, Rabi frequency $\alpha$ ) to create a long-lived coherence between states $|\mathrm{g}\rangle$ and $|\mathrm{s}\rangle$ [1, as depicted in Fig. 1(a), producing a narrow symmetric transparency peak in the spectrum of the signal field near the two-photon resonance $\left(\nu-\nu_{\mathrm{C}} \approx \omega_{g s}\right)$. Simultaneously, the signal field experiences a steep variation in refractive index, thereby reducing the pulse's group velocity ("slow light"), and leading to a pulse delay time of $\tau=(d \gamma) /|\Omega|^{2} \gg L / c[3]$, where $\gamma$ is the optical polarization decay rate.

While this description is sufficient in the limit of low optical depth [17, at high optical depth, accompanying nonlinear processes become important. A sufficiently strong control field can excite the far off-resontant transition from $|\mathrm{g}\rangle$ to $|\mathrm{e}\rangle$ [15], and generate a Stokes field $\alpha^{\prime}$ via the four-wave mixing (FWM) process. We theoretically model this effect by considering a double- $\Lambda$ configuration, consisting of a near-resonant $\Lambda$ system formed by the control and signal fields and of the additional far-detunied $\Lambda$ system formed by the same control field $\left(\nu_{\mathrm{C}}=\omega_{e s}\right.$, Rabi frequency $\Omega^{\prime}$ ), applied to the state $|\mathrm{g}\rangle$ and by an additional Stokes field $\left(\nu^{\prime}=\omega_{e s}-\Delta_{\mathrm{hf}}-\delta\right.$, Rabi frequency $\alpha^{\prime}$ ). In such system, we can use Floquet theory [23] to adiabatically eliminate the off-resonant interaction via $\Omega^{\prime}$ and $\alpha^{\prime}$. As a result, to first order in $1 / \Delta_{\mathrm{hf}}$ and in $\alpha^{\prime}$, one obtains an effective Rabi frequency $\Omega^{\prime} \alpha^{\prime *} / \Delta_{\mathrm{hf}}$ coupling $|\mathrm{g}\rangle$ and $|\mathrm{s}\rangle$, while the states $|\mathrm{e}\rangle$ and $|\mathrm{g}\rangle$ acquire small light shifts $\delta_{s}=\left|\Omega^{\prime}\right|^{2} / \Delta_{\mathrm{hf}}$ and $-\delta_{s}$, respectively. For our Clebsch-Gordan coefficients [17, $\left|\Omega^{\prime}\right|^{2}=3|\Omega|^{2}$.

The rotating-frame Hamiltonian describing this interaction is:

$$
\begin{aligned}
\hat{H}= & -\left(\delta-\delta_{s}\right)|\mathrm{s}\rangle\left\langle\mathrm{s}\left|-\left(\delta-2 \delta_{s}\right)\right| \mathrm{e}\right\rangle\langle\mathrm{e}| \\
& -\left[\alpha|\mathrm{e}\rangle\langle\mathrm{g}|+\Omega| \mathrm{e}\rangle\left\langle\mathrm{s}\left|+\frac{\Omega^{\prime} \alpha^{\prime *}}{\Delta_{\mathrm{hf}}}\right| \mathrm{s}\right\rangle\langle\mathrm{g}|+\text { H.c. }\right] .
\end{aligned}
$$

Here, the Rabi frequencies of the signal and the Stokes fields are $\alpha=\mathcal{E} \mu / \hbar$ and $\alpha^{\prime}=\mathcal{E}^{\prime} \mu^{\prime} / \hbar$, where $\mathcal{E}$ and $\mathcal{E}^{\prime}$ are 
the corresponding slowly-varying envelopes, and $\mu$ and $\mu^{\prime}$ are the (real) dipole matrix elements of the respective transitions.

In the undepleted pump and adiabatic approximations, the Fourier components of the signal field $\mathcal{E}(\omega)$ and the Stokes field $\mathcal{E}^{\prime}(\omega)$ propagate according to the coupled differential equations, to linear order in $\alpha$ and $\alpha^{\prime}$ [15, 16, 24]:

$$
\partial_{z}\left[\begin{array}{c}
\mathcal{E}(z, \omega) \\
\mathcal{E}^{\prime *}(z, \omega)
\end{array}\right]=i \frac{d \gamma}{F L}\left[\begin{array}{cc}
\delta-\delta_{s}+\omega+i \gamma_{0} & -\Omega^{2} / \Delta_{\mathrm{hf}} \\
\Omega^{* 2} / \Delta_{\mathrm{hf}} & 0
\end{array}\right]\left[\begin{array}{c}
\mathcal{E}(z, \omega) \\
\mathcal{E}^{\prime *}(z, \omega)
\end{array}\right]
$$

where we take into account the optical polarization decay rate $\gamma$ and the ground state decay rate $\gamma_{0} \approx \gamma_{s g}+\gamma \frac{\left|\Omega^{\prime}\right|^{2}}{\Delta_{\mathrm{hf}}^{2}}$ $[25]$, and have set $F=|\Omega|^{2}+\left[\gamma-i\left(\delta-2 \delta_{s}+\omega\right)\right]\left[\gamma_{0}-\right.$ $\left.i\left(\delta-\delta_{s}+\omega\right)\right]$. Under the assumption that $\Omega$ is real and z-independent, Eq. (2) can be solved analytically for $\mathcal{E}(z, \omega)$ and $\mathcal{E}^{\prime *}(z, \omega)$, for the conditions corresponding to our experiment: $\mathcal{E}^{\prime *}(0, \omega)=-f \mathcal{E}(0, \omega)$ : the Stokes seed has the same initial temporal lineshape as the sig- nal pulse, but with an opposite phase and with some amplitude scaling factor $0<f \leq 1$. Defining the Raman detuning as $\Delta_{\mathrm{R}}=-\Omega^{2} / \Delta_{\mathrm{hf}}$, and with $\beta(\omega) \equiv$ $\sqrt{\left[\gamma_{0}-i\left(\delta-\delta_{s}+\omega\right)\right]^{2}+4 \Delta_{\mathrm{R}}^{2}}, \sigma(\omega) \equiv \frac{1}{2} \frac{d \gamma}{F L}\left(\delta-\delta_{s}+\omega+\right.$ $\left.i \gamma_{0}\right)$, and $\xi(\omega) \equiv \frac{1}{2} \frac{d \gamma}{F L} \beta(\omega)$, we find the following analytic expressions for the Fourier components of the signal and Stokes fields [16, 26]:

$$
\begin{aligned}
\mathcal{E}(z, \omega) & =\mathcal{E}(0, \omega) \mathrm{e}^{i \sigma(\omega) z}\left[\cosh [\xi(\omega) z]+i\left(\frac{\sigma(\omega)}{\xi(\omega)}-f \frac{2 \Delta_{\mathrm{R}}}{\beta(\omega)}\right) \sinh [\xi(\omega) z]\right], \\
\mathcal{E}^{\prime *}(z, \omega) & =-f \mathcal{E}(0, \omega) \mathrm{e}^{i \sigma(\omega) z}\left[\cosh [\xi(\omega) z]-i\left(\frac{\sigma(\omega)}{\xi(\omega)}-\frac{1}{f} \frac{2 \Delta_{\mathrm{R}}}{\beta(\omega)}\right) \sinh [\xi(\omega) z]\right] .
\end{aligned}
$$

Eqs. (3) and (4) fully describe the propagation of the light fields through the atomic medium. Theoretically, the measured transmission spectra of the signal and Stokes fields are computed as $|\mathcal{E}(L)|$ and $\left|\mathcal{E}^{\prime}(L)\right|$, respectively using Eqs. (3) and (4). We interpret equations (3) and (4) by first applying a few simplifications, similar to Ref. [26], by first shifting the two-photon detuning by the light shift, defining $\widetilde{\delta}=\delta-\delta_{s}$ ( setting $\omega=0$ for continuous wave measurements), and considering large $|\widetilde{\delta}| \gg 2\left|\Delta_{\mathrm{R}}\right|$, but also $|\widetilde{\delta}| \ll \Omega$, and $|\widetilde{\delta}| \gg \gamma_{0}$. Under these assumptions, $\beta \approx i \widetilde{\delta}$, and for our parameters, $2 \xi L=2 i \sigma L \approx i \frac{\widetilde{\delta}}{\Omega^{2} /(d \gamma)}-\frac{\widetilde{\delta}^{2}}{\left[\Omega^{2} /(\sqrt{d} \gamma)\right]^{2}}$, where the denominators of the two terms represent, respectively, the inverse of the EIT group delay and the square of the width of the EIT transparency window. Under these approximations, which hold well in our experiments, the signal and Stokes amplitudes after the cell are:

$$
\begin{aligned}
|\mathcal{E}(L)| & =\mathcal{E}(0)\left|e^{2 i \sigma L}-f \frac{\Omega^{2}}{\Delta_{\mathrm{hf}} \widetilde{\delta}}\left(1-e^{2 i \sigma L}\right)\right|, \\
\left|\mathcal{E}^{\prime}(L)\right| & =\mathcal{E}(0)\left|\frac{\Omega^{2}}{\Delta_{\mathrm{hf}} \widetilde{\delta}}\left(1-e^{2 i \sigma L}\right)-f\right| .
\end{aligned}
$$

These expressions allow us to interpret the transmission spectra for both fields in terms of an interference between EIT and FWM effects, where the FWM effect is represented by the terms proportional to $1 / \Delta_{\mathrm{hf}}[26$. At small optical depth, the effect of FWM on the signal field transmission is negligible, and we observe a typical symmetric EIT transmission peak (not shown). As optical depth increases, the FWM term $\left(\propto 1 / \Delta_{\mathrm{hf}}\right)$ in Eq. (5) becomes more noticeable relative to the EIT term, which reduces with $d$ due to the narrowing of the EIT window. Since the phase of $e^{2 i \sigma L}$ in Eq. 5 is $\approx \widetilde{\delta} /\left(v_{g} / L\right)$, for $\widetilde{\delta}>0, \widetilde{\delta}=n \pi v_{g} / L$ gives destructive interference (and hence dips in the spectrum) for even $n$, and constructive interference for odd $n$. For $\widetilde{\delta}<0$, the opposite case is true: even $n$ yields constructive interference; odd $n$ yields destructive interference.

\section{SPECTRAL MEASUREMENTS}

We record the transmission spectra for continuous signal and Stokes fields by sweeping the EOM frequency, which simultaneously changes the two-photon detuning $\delta$, and measuring their amplitude variations after the cell. Fig. 2(a,b) depicts the experimental results for an optical depth of $2 d=52$. Solid lines represent the spectra corresponding to the signal field transmission; dashed 

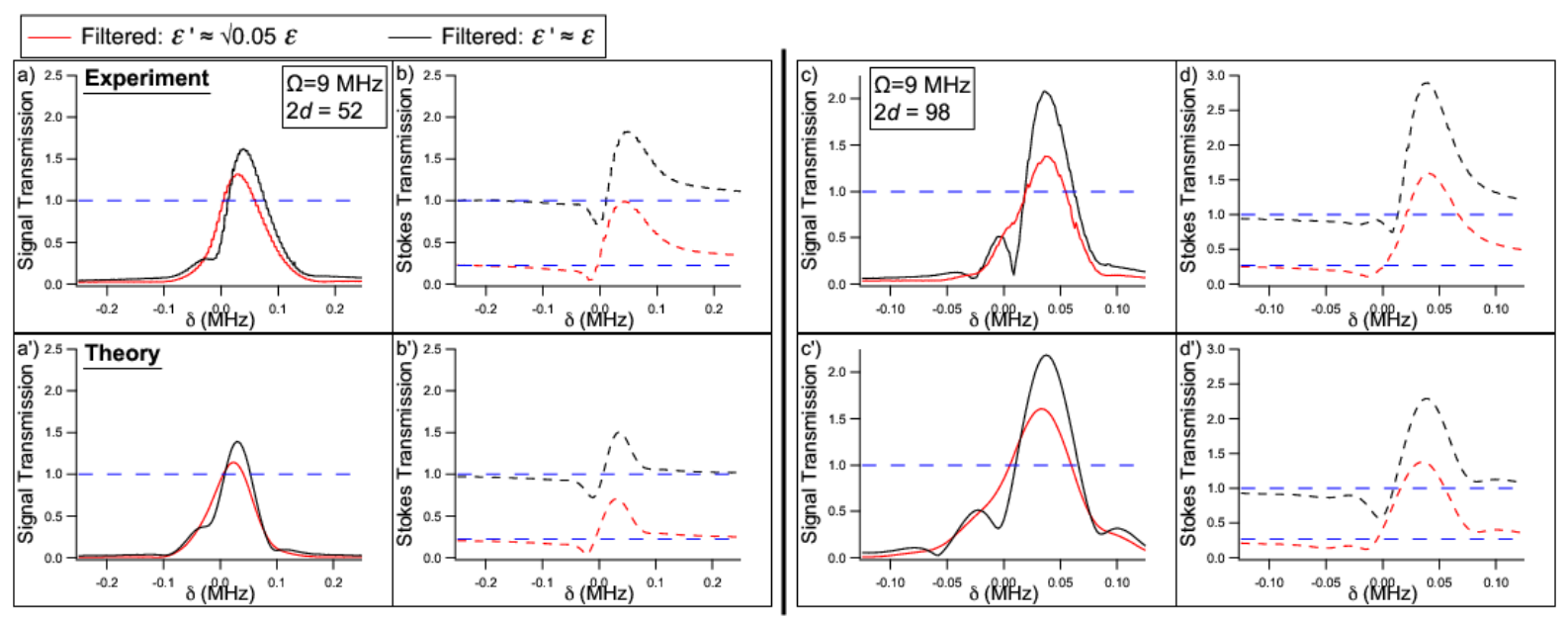

FIG. 2: (Color Online) (a, b) Signal and stokes amplitude spectra at an optical depth of $2 d=52$ with a beam diameter of $4 \mathrm{~mm}$ and $\Omega / 2 \pi=9 \mathrm{MHz}$. Black traces are with a full Stokes seed present. Red traces are with the Stokes seed amplitude attenuated to $\approx \sqrt{0.05}$ of the signal field's amplitude. $\left(\mathrm{a}^{\prime}, \mathrm{b}^{\prime}\right)$ Corresponding theoretical predictions. (c, d) Signal and stokes amplitude spectra at an optical depth of $2 d=98$. $\left(\mathrm{c}^{\prime}, \mathrm{d}^{\prime}\right)$ Corresponding theoretical predictions.
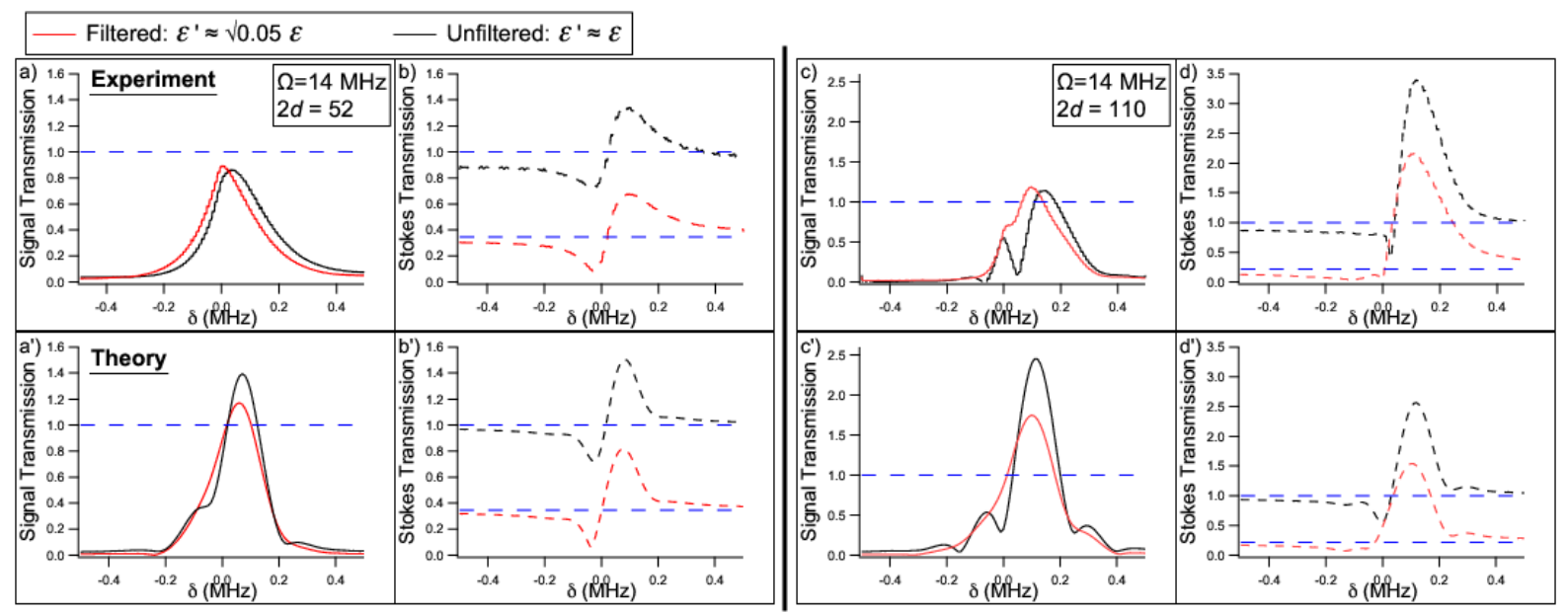

FIG. 3: (Color Online) (a, b) Signal and stokes amplitude spectra at an optical depth of $2 d=52$ with a beam diameter of 2.6 $\mathrm{mm}$ and $\Omega / 2 \pi=14 \mathrm{MHz}$. Black traces are with a full Stokes seed present. Red traces are with the Stokes seed amplitude attenuated to $\approx \sqrt{0.05}$ of the signal field's amplitude. $\left(\mathrm{a}^{\prime}, \mathrm{b}^{\prime}\right)$ Corresponding theoretical predictions. (c, d) Signal and stokes amplitude spectra at an optical depth of $2 d=110$. $\left(\mathrm{c}^{\prime}, \mathrm{d}^{\prime}\right)$ Corresponding theoretical predictions.

lines correspond to the Stokes field transmission spectra. Black lines are with no Stokes filtering (i.e., $f=1$ ); red lines are with a Stokes intensity attenuation so that $f=\sqrt{0.05}$. These two values of $f(1$ and $\sqrt{0.05})$ are shown by horizontal dashed blue lines in Figs. $2\left(b, b^{\prime}\right)$ and represent the input Stokes amplitude. With the reduced Stokes seed (red curves), the effects of FWM are suppressed, and we observe a slightly amplified and nearly symmetric EIT transmission peak. However, when the full Stokes seed field is present (black curves), on one hand we observe more gain in the signal field, but on the other hand the FWM/EIT destructive interference be- comes more evident by the presence of a "knee" in the signal spectra for small negative detunings. These results are in very good agreement with the predictions of the theory [see Fig. (2) $\left(\mathrm{a}^{\prime}, \mathrm{b}^{\prime}\right)$ ], which are calculated from the full expressions in Eqs. (3) and (4) with no free parameters, where $\Omega, \gamma_{0}, d$, and $\gamma$ were computed as in Ref. [17.

Spectra taken at higher optical depth reveal more clear evidence of the constructive and destructive interference between EIT and FWM. Fig.22(c,d) presents similar spectra recorded for the same signal and Stokes fields, but at an optical depth of $2 d=98$. In the case of no 
Stokes attenuation (black curves), the theoretical curve in Fig. 2 $2\left(c^{\prime}\right)$ exhibits, as expected, destructive interference at $\delta=\delta-\delta_{s}=n \pi v_{g} / L$ for $n=-3,-1$, and 2 [here $\pi v_{g} / L=(2 \pi) 31 \mathrm{kHz}$ and $\delta_{s}=(2 \pi) 36 \mathrm{kHz}$. While slightly shifted, these three points of destructive interference are also clearly visible in the experimental measurement of Fig. 2(c). Even when the Stokes seed field is suppressed, its presence leads to significant distortions in the signal transmission resonance.

We repeated similar spectral measurements after reducing the diameter of the beam by a factor of 1.5 , which increased the control field Rabi frequency to $\Omega / 2 \pi=14$ $\mathrm{MHz}$, and corresponded to a larger light shift of $\delta_{s} / 2 \pi \approx$ $85 \mathrm{kHz}$. Larger control intensity and smaller beam size allowed us to reduce the control field absorption at high optical depths and stay within the theoretical model's approximations. Fig. 3 shows the experimental and corresponding theoretical spectra for optical depths of 52 and 110. The larger Rabi frequency results in a larger $v_{g}$ than above, and thus more closely spaced spectral dips and peaks. There is an excellent agreement between the experiment and the theory for the Stokes spectra, and for the signal spectra at negative detuning. However, the theoretical model for signal transmission diverges from experimental observations at positive detuning, indicating the presence of some unaccounted mechanisms such as nonunity control field refractive index, atomic diffusion [27], and/or the effects of the multi-level structure of the atoms.

\section{SLOW LIGHT MEASUREMENTS}

In this section we discuss the slow light regime for the signal field pulses in the presence of the co-propagating seeded Stokes field. In particular, we are interested in the prospect of manipulating the signal pulse group delay and amplitude via the controllable amplitude of the input Stokes field. It is convenient to use Eqs. (3), (4) to analyze the dynamics of each pulse propagation through the medium by calculating the variation in the complex amplitudes of both fields for each spectral component of the input pulse and then by Fourier transforming the resulting expressions back into the temporal domain. The group delay of the signal field is determined from the acquired phase, which consists of two contributions. The first from the first exponential in Eq.(3), and it is the same for all spectral components of the pulse: $\tau_{0}=\frac{d}{d \omega} \operatorname{Re}[\sigma(\omega) z] \approx \frac{d \gamma z}{2 L \Omega^{2}}$. Notably, this value is exactly half of the pulse delay expected from the pure EIT system. The second contribution is from the expression in brackets in Eq. (3). The value of this additional delay depends explicitly on the detuning of the signal pulse from resonance and may vary significantly for different spectral components of the pulse. Below we discussed three distinct scenarios for the pulse two-photon detuning: $\delta=2\left|\Delta_{\mathrm{R}}\right|, \delta=2 \delta_{s}$, and $\delta=0$.

Figs. $4(\mathrm{a}, \mathrm{b})$ and $5(\mathrm{a}, \mathrm{b})$ correspondingly present the experimental data for $6 \mu$ s-long (FWHM) signal and Stokes pulses (which corresponds to a bandwidth of $\pm(2 \pi) 31 \mathrm{kHz}$ around the carrier frequency) when the full Stokes field is present, and when the Stokes field is suppressed. Respective graphs $\left(a^{\prime}\right)$ and $\left(b^{\prime}\right)$ give the prediction of the calculations based on the complete solutions of Eqs. (3), (4). In these calculations, we use a control field with Rabi frequency $\Omega / 2 \pi=14 \mathrm{MHz}$, corresponding to $\delta_{s} / 2 \pi=84 \mathrm{kHz}$ and $\Delta_{\mathrm{R}} / 2 \pi=-28 \mathrm{kHz}$.

For more insight into the spectral dynamics of the pulse, we also plot the calculated time delay experienced by the signal field spectral components $\omega$ [Figs. 4 (c) and $5(\mathrm{c})]$, and the spectral gain $|\mathcal{E}(\omega, L)| /|\mathcal{E}(\omega, 0)|$ [Figs. $4(\mathrm{~d})$ and [5(d)]. These last graphs also show the spectral bandwidth of the input pulse (the blue, dashed curve) for reference.

Case I: $\delta=2\left|\Delta_{\mathrm{R}}\right|$

The data corresponding to the case $\delta=2\left|\Delta_{\mathrm{R}}\right|=$ $(2 \pi) 56 \mathrm{kHz}$ are shown in Figs. 4 and 5 in red. Although the theoretical calculations predict only a weak dependence of the signal pulse on the amplitude of the Stokes seed, the experimental data show much stronger dependence: for the full seeded Stokes field the experimental pulse shows small delay and noticeable attenuation, while when the Stokes field is small, the signal pulse experiences some small gain and much larger delay. The latter is much closer to the theoretical expectations of gain of $\approx 1.5$ and the delay of $\approx 6 \mu$ s. The experimental results for the Stokes field, however, match the theory much more accurately, which may indicate that the absorption of the signal field is underrepresented by the model.

The analysis of the spectral gain and delay for both cases $(f=1$ and $f=\sqrt{0.05})$ provides some qualitative understanding of the observed pulse behavior. For the case of the full Stokes field, Fig. 4(c) shows that all the signal spectral components with $\omega>0$ experience a roughly uniform delay of $\approx 5 \mu \mathrm{s}$, whereas the components $\omega<0$ experience a longer delay, resulting in the pulse spreading as it traverses the cell. However, this effect is somewhat suppressed by lower spectral gain for $\omega<0$ (Fig. 4d). With the Stokes field attenuated [Fig. 5(c,d)], we expect that all signal spectral components $\omega$ experience a uniform delay of $\approx 6 \mu \mathrm{s}$, and a slight gain, resulting in delayed propagation with little pulseshape distortion, as corroborated well in Fig. 5(a).

Case II: $\delta=2 \delta_{s}$

The green curves in Figs. 4 and 5 depict the results of slow light experiments with a two-photon detuning of $\delta=2 \delta_{s}=(2 \pi) 168 \mathrm{kHz}$. Figs. 4(c,d) illustrate that for the unfiltered Stokes field $(f=1)$, all frequency components of the initial signal pulse experience a nearly identical delay of $\approx 5 \mu \mathrm{s}$ - indicating very little pulse spread. Simultaneously, the central component should be amplified by a factor of $\approx 1.8$. This prediction matches well with both the experimental [Figs. [4(a,b)] and theoretical [Figs. $\left.4\left(a^{\prime}, b^{\prime}\right)\right]$ pulses. When the Stokes seed is attenuated $(f=\sqrt{0.05})$, the signal pulse experiences a slightly 


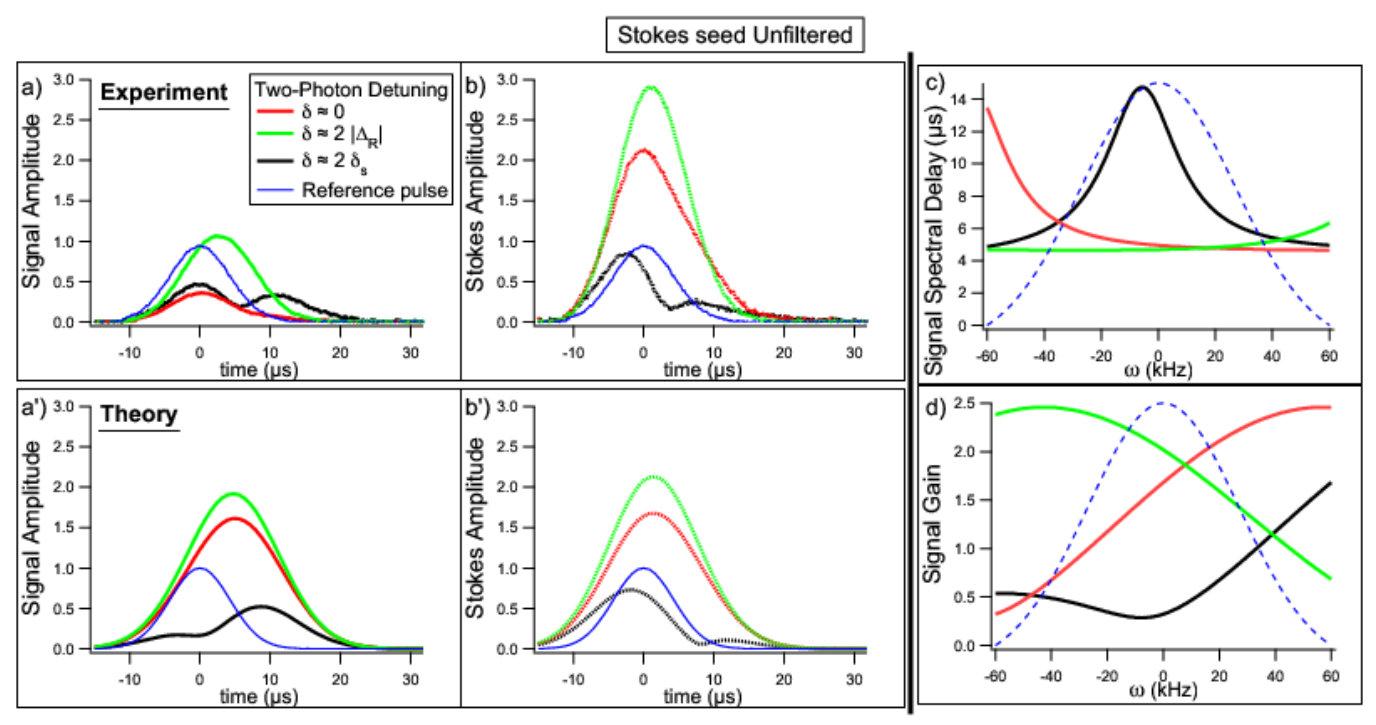

FIG. 4: (Color Online) (a) Slow light on the signal channel at an optical depth of $2 d=110$ with no Stokes seed attenuation (i.e., $f=1$ ) for signal detunings $\delta=2\left|\Delta_{\mathrm{R}}\right|$ (red curves), $\delta=2 \delta_{s}$ (green curves), and $\delta=0$ (black curves). The thin blue curve is the initial reference pulse. (b) Corresponding Stokes channel. ( $\left.\mathrm{a}^{\prime}, \mathrm{b}^{\prime}\right)$ Corresponding theoretical predictions from Eqs (3), 44). (c) Theoretical total delay dispersion experienced by the signal pulse frequency components after traversing length $L$. The blue dashed curve depicts the frequency spread of the input signal pulse. (d) Predicted signal gain dispersion.

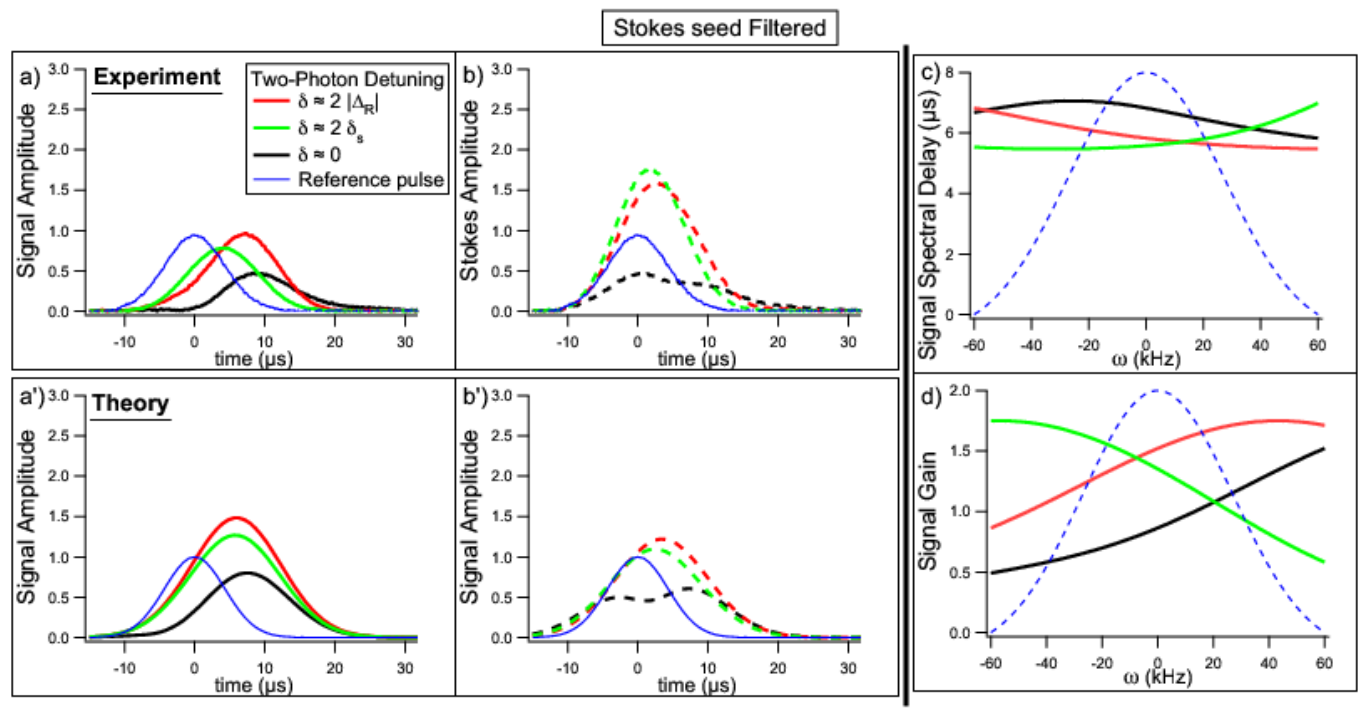

FIG. 5: (Color Online) Same as Fig. 4 , but with Stokes seed attenuation $(f=\sqrt{0.05})$.

longer delay of $\approx 5.8 \mu \mathrm{s}$, but will also be less amplified, according to Fig. 5(d). The experimental result [green curve in Fig. 5(a) reproduces this predicted delay, but shows a small attenuation rather than gain, possibly indicating the presence of an additional decay mechanism.

Case III: $\delta=0$ The black curves in Figs. 4 and 5 depict the results of slow light experiments with a twophoton detuning of $\delta=0$. This case most clearly demonstrates the merits of Stokes seed attenuation. For the unfiltered Stokes seed $f=1$, different spectral compo- nents will acquire very different phase and gain while propagating through the interaction region. In particular, Fig 4(c) shows large variation in the spectral delay - from $14 \mu \mathrm{s}$ for central frequencies to $5 \mu \mathrm{s}$ for the farther detuned components. When combined with the gain curve, shown in Fig. 4(d), such variations should greatly distort the shape of the output pulses. In fact, the expected output closely resembles a double-peaked pulse, and is quite similar to that observed in the experiment. When the Stokes seed is filtered $(f=\sqrt{0.05})$, as in Fig. 5 . 
the differential delay is suppressed, and all spectral components experience a common delay of nearly $7 \mu \mathrm{s}$, but at the sacrifice of gain, which is $<1$. Figs. 5 (a,b) show excellent agreement with the corresponding theoretical curves.

\section{CONCLUSIONS}

In conclusion, we have demonstrated that both steadystate and dynamic properties of the signal field propagating under the EIT conditions are strongly effected by resonant four-wave mixing that arise under the conditions of EIT at high optical depth. This process is well- modeled by a simple double- $\Lambda$ system, where the output signal and stokes field amplitudes are the results of interference of "traditional" EIT and FWM. We have shown that by attenuating the amplitude of the seeded Stokes field, we can partially control the optical properties of the medium for the signal field. Moreover, by adjusting the central frequency of the input signal field around two-photon resonance, in the presence of Stokes seed field we can achieve longer pulse delay and/or amplification of the signal pulse.

The authors thank M. D. Lukin for useful discussions. This research was supported by NSF grant PHY0758010, Jeffress Research grant J-847, and the College of William \& Mary.
[1] M. D. Lukin, Rev. Mod. Phys. 75, 457 (2003).

[2] M. Fleischhauer, A. Imamoglu, and J. P. Marangos, Rev. Mod. Phys. 77, 633 (2005).

[3] R. W. Boyd, D. J. Gauthier, Progress in Opt. 43, 497 (2002).

[4] M. Fleischhauer, and M. D. Lukin, Phys. Rev. Lett. 84, 5094, (2000); Phys. Rev. A 65, 022314 (2002).

[5] L. M. Duan, M. D. Lukin, J. I. Cirac, and P. Zoller, Nature 414, 413 (2001).

[6] J. Simon, H. Tanji, J. K. Thompson, V. Vuletic, Phys. Rev. Lett. 98, 183601 (2007).

[7] C. A. Muschik, I. de Vega, D. Porras, J. I. Cirac, Phys. Rev. Lett. 100, 063601 (2008).

[8] R. S. Tucker, P. C. Ku, C. J. Chang-Hasnain, J. Lightwave Tech. 23, 4046 (2005).

[9] R. W. Boyd, D. J. Gauthier, A. L. Gaeta, A. E. Willner A. E., Phys. Rev. A 71, 023801 (2005).

[10] A. V. Gorshkov, A. André, M. Fleischhauer, A. S. Sørensen, and M. D. Lukin, Phys. Rev. Lett. 98, 123601 (2007).

[11] H. Kang, G. Hernandez, and Y. Zhu, Phys. Rev. A 70, 061804(R) (2004).

[12] V. Wong, R. S. Bennink, A. M. Marino, R. W. Boyd, C. R. Stroud, and F. A. Narducci, Phys. Rev. A 70, 053811 (2004).

[13] K. I. Harada, T. Kanbashi, M. Mitsunaga, and K. Motomura, Phys. Rev. A 73, 013807 (2006).

[14] G. S. Agarwal, T. N. Dey, and D. J. Gauthier, Phys. Rev.
A 74, 043805 (2006).

[15] M. D. Lukin, P. R. Hemmer, M. Löffler, and M. O. Scully, Phys. Rev. Lett. 81, 2675 (1998).

[16] M. D. Lukin M. D., A. B. Matsko, M. Fleischhauer, M. O. Scully, Phys. Rev. Lett. 82, 1847 (1999).

[17] N. B. Phillips, A. V. Gorshkov, I. Novikova, Phys. Rev. A 78, 023801 (2009).

[18] V. Boyer, A. M. Marino, R. C. Pooser, P. D. Lett, Science, 5888, 544 (2008).

[19] A. B. Matsko, D. V. Strekalov, L. Maleki, Opt. Express, 13, 2210 (2005).

[20] R. M. Camacho, P. K. Vudyasetu, J. C. Howell, J. Nature Photonics, 3, 103 (2009).

[21] M. D. Rotondaro and G. P. Perram, Phys. Rev. A 58, 2023 (1998).

[22] As in Ref. [17, we define the Rabi frequency $\Omega$ as $|\Omega|^{2}=$ $\wp_{e s}^{2} I /\left(2 \hbar^{2} \varepsilon_{0} c\right)$, where $I$ is the control field intensity.

[23] K. Drese, M. Holthaus, Eur. Phys. J. D, 5, 119 (1999).

[24] M. D. Lukin, M. Fleischhauer, A. S. Zibrov, H. G. Robinson, V. L. Velichansky, L. Hollberg, and M. O. Scully, Phys. Rev. Lett. 79, 2959 (1997).

[25] André A. Ph.D. Thesis. Harvard University, 2005.

[26] T. Hong, A. V. Gorshkov, D. Patterson, A. S. Zibrov, J. M. Doyle, M. D. Lukin, M. G. Prentiss, Phys. Rev. A, 79, 013806 (2009).

[27] O. Firstenberg, M. Shuker, N. Davidson, A. Ron, Phys. Rev. Lett., 102, 043601 (2009). 